Visual hallucinations in dementia with Lewy bodies: transcranial magnetic stimulation study

John-Paul Taylor, Michael Firbank, Nicola Barnett, Sarah Pearce, Anthea Livingstone, Urs Mosimann, Janet Eyre, lan G. McKeith and John T. O'Brien

BJP 2011, 199:492-500.

Access the most recent version at DOI: 10.1192/bjp.bp.110.090373

References This article cites 0 articles, 0 of which you can access for free at: http://bjp.rcpsych.org/content/199/6/492\#BIBL

Reprints/ To obtain reprints or permission to reproduce material from this paper, please permissions write to permissions@rcpsych.ac.uk

You can respond http://bjp.rcpsych.org/cgi/eletter-submit/199/6/492

to this article at

Downloaded http://bjp.rcpsych.org/ on February 18, 2014

from Published by The Royal College of Psychiatrists 


\section{Visual hallucinations in dementia with Lewy bodies: transcranial magnetic stimulation study}

John-Paul Taylor, Michael Firbank, Nicola Barnett, Sarah Pearce, Anthea Livingstone, Urs Mosimann, Janet Eyre, Ian G. McKeith and John T. O’Brien

\section{Background}

The aetiology of visual hallucinations is poorly understood in dementia with Lewy bodies. Pathological alterations in visual cortical excitability may be one contributory mechanism.

\begin{abstract}
Aims
To determine visual cortical excitability in people with dementia with Lewy bodies compared with aged-matched controls and also the relationship between visual cortical excitability and visual hallucinations in dementia with Lewy bodies.

\section{Method}

Visual cortical excitability was determined by using transcranial magnetic stimulation (TMS) applied to the occiput to elicit phosphenes (transient subjective visual responses) in 21 patients with dementia with Lewy bodies and 19 age-matched controls.
\end{abstract}

\section{Results}

Phosphene parameters were similar between both groups However, in the patients with dementia with Lewy bodies, TMS measures of visual cortical excitability correlated strongly with the severity of visual hallucinations $(P=0.005)$ Six patients with dementia with Lewy bodies experienced visual hallucination-like phosphenes (for example, seeing people or figures on stimulation) compared with none of the controls $(P=0.02)$.

\section{Conclusions}

Increased visual cortical excitability in dementia with Lewy bodies does not appear to explain visual hallucinations but it may be a marker for their severity.

\section{Declaration of interest}

None.
Visual hallucinations are a feature of many psychiatric disorders, including schizophrenia and bipolar disorder as well as prototypical conditions such as Charles Bonnet syndrome. However, their prevalence is particularly high in Lewy body diseases including Parkinson's disease, Parkinson's disease with dementia and dementia with Lewy bodies; and in the latter up to $80 \%$ of individuals are affected. ${ }^{1}$ Untreated, visual hallucinations in dementia with Lewy bodies can markedly impair quality of life ${ }^{2}$ and significantly increase caregiver distress. ${ }^{3}$ However, although a number of causative models for complex visual hallucinations have been proposed (see, for example, Collerton et $a{ }^{4}{ }^{4}$ Diederich et $a l^{5}{ }^{5}$ ffytche et $a l^{6}$ and Manford \& Andermann ${ }^{7}$ ) no specific model has yet been proved definitive. Deafferentation models of visual hallucinations, favoured in conditions such as Charles Bonnet syndrome, suggest that defective sensory input from the eye to the visual cortex can lead to the 'release' of visual cortical areas and subsequent visual hallucinations. ${ }^{6,7}$ A similar deafferentation process leading to hyperexcitability of the visual cortex could also occur in dementia with Lewy bodies; certainly across the Lewy body disease spectrum there is evidence for pregeniculate dysfunction, including disturbances in retinal dopaminergic circuitry, altered inter-retinal neural transmission and neuropathological abnormalities in photoreceptors and outer plexiform layers (for review, see Archibald et $a l^{8}$ ).

Others have speculated that dysfunction in association areas or impaired attentional binding is crucial to visual hallucination formation in Lewy body disease. ${ }^{4,9}$ In dementia with Lewy bodies, Lewy bodies in the inferior temporal cortex at autopsy have been linked with visual hallucinations during life, ${ }^{10}$ there is extensive parietal hypoperfusion ${ }^{11}$ and significant glucose hypometabolism in visual association areas; ${ }^{12}$ these abnormalities combined with posterior white matter disruption ${ }^{12,13}$ may lead to perturbations in visual processing with disturbances in afferent inputs and recurrent feedback loops. In support of this, a functional magnetic resonance imaging (fMRI) study examining visual object processing ${ }^{9}$ found that people with Parkinson's disease with visual hallucinations had reduced blood oxygen level-dependent activation in lateral occipital and extrastriate temporal areas compared with individuals with Parkinson's disease who did not hallucinate prior to image recognition. The authors argued that this favoured the role of impaired bottom-up processing from early visual areas to association areas in the pathogenesis of visual hallucinations.

One way to provide further insights into the aetiology of visual hallucinations in dementia with Lewy bodies is to investigate whether cortical visual excitability, i.e. the responsiveness of visual cortex to external stimuli, is altered, and one method of probing cortical excitability is through occipital transcranial magnetic stimulation (TMS). This is a non-invasive method of brain stimulation, which when applied over the occipital cortex produces transient visual percepts called phosphenes. The origin of phosphenes is likely to be within the superficial dorsal occipital lobe since the current density generated by TMS dissipates with the square of the distance. Thus phosphenes have been presumed to arise from either direct excitation of neurons within the visual cortex or from extracalcarine sites, although there is more consistent evidence that phosphenes originate directly from excitation of the termination of the optic radiation in Brodmann area 17 and excitation of back-projecting fibres from Brodmann area 18/19. ${ }^{14}$ However, regardless of stimulation site an intact striate visual cortex is required for the perception of phosphenes ${ }^{15,16}$ and the phosphene threshold, the stimulation intensity level at which phosphenes are just perceived, is a reliable marker of the excitability of the visual cortex. $^{17,18}$

A number of studies have shown in dementia with Lewy bodies that significant hypoperfusion and impaired glucose metabolism occur in the occipital pole and these observations would intuitively favour decreased early visual cortical processing/excitability. Nevertheless, although these findings have 
been linked by some to the presence of visual hallucinations ${ }^{19,20}$ this has not been a consistent finding, with other studies demonstrating that occipital perfusion can be normal in dementia with Lewy bodies. ${ }^{21}$ In addition, O'Brien et $a l^{22}$ found a relationship between nicotinic receptor binding in primary and secondary visual cortices and visual hallucinations in people with dementia with Lewy bodies, but a lack of association with occipital perfusion deficits, suggesting that significant functional receptor changes can occur in the absence of obvious alterations in perfusion. This, with the lack of observed neuropathology in the occipital pole ${ }^{23}$ in dementia with Lewy bodies, would favour the argument that the visual cortex is 'structurally' intact in dementia with Lewy bodies and thus reduced visual excitability/ increased phosphene threshold is unlikely. The phosphene threshold is decreased by factors such as light deprivation, ${ }^{24}$ pregeniculate blindness ${ }^{18}$ and migraine; $;^{25}$ these are all visual cortical hyperexcitable states that are often associated with visual hallucinations. Certainly reduced phosphene thresholds have been observed in heavy users of ecstasy (3,4-methylenedioxymethamphetamine) who have visual hallucinations. ${ }^{26}$ Thus in the present study we hypothesised that it would be likely that: (a) phosphene thresholds would be lower rather than higher in patients with dementia with Lewy bodies (patients with DLB) with visual hallucinations compared with controls (healthy controls); (b) in patients with DLB, lower phosphene thresholds would be associated with more frequent and severe visual hallucinations.

\section{Method}

\section{Participants}

Twenty-one patients with DLB who had experienced visual hallucinations at least once in the month before TMS testing were recruited from a local community-dwelling population of patients in the North-East of England. Nineteen age-matched controls were selected from friends and spouses of patients included in this and previous studies. The study was approved by the local ethics committee. The patients with DLB underwent detailed physical, neurological and neuropsychiatric examination. The diagnosis of dementia with Lewy bodies was made independently by two experienced senior clinicians using the revised International Consensus Guidelines for dementia with Lewy bodies ${ }^{27}$ and all study patients met criteria for probable dementia with Lewy bodies.

All control and 18 of the 21 patients with DLB underwent structural $T_{1}$-weighted magnetic resonance imaging (MRI). Cognitive function in all participants was assessed using the Cambridge Cognitive Examination ${ }^{28}$ (CAMCOG, maximum score 105) and the Mini-Mental State Examination ${ }^{29}$ (MMSE, maximum score 30 ). The presence and severity of any extrapyramidal signs were graded using the motor component of the Unified Parkinson's Disease Rating Scale (UPDRS). ${ }^{30}$ The controls demonstrated no evidence of dementia (from history and score $>80$ on CAMCOG). Exclusion criteria for all participants included contraindications for MRI or TMS, severe visual impairment, history of alcohol/substance misuse, use of benzodiazepine or anticonvulsant medication, past neurological or psychiatric history (apart from dementia), focal brain lesions on brain imaging or the presence of other severe or unstable medical illness. Patients with DLB were required to have a primary caregiver who had regular daily contact with the patient.

All participants had measurement of their best near visual acuity on Landolt broken rings or Snellen chart (test distance $40 \mathrm{~cm}$ ) after correction of any refractive errors. Participants were only included in the study if they had no visual field defects and intact ocular movements on neurological examination.
Assessment of visuoperceptual function was carried out using previously described angle discrimination and overlapping figures tasks. ${ }^{31}$ Participants underwent 20 trials for each task and a visuoperceptual score was derived (sum of scores) for further analysis.

Prior to TMS testing in the patients with DLB, the primary caregiver completed the Clinician Assessment of Fluctuation (CAF) scale and the One Day Fluctuation Assessment Scale (ODFAS). ${ }^{32}$ Clinically if patients demonstrated that they had a new and sudden onset of marked cognitive fluctuations in the $24 \mathrm{~h}$ prior to TMS testing, which could be as a result of factors such as an acute illness (for example, urinary tract infection) or delirium, they were not tested until they returned to their prior clinical state to avoid the potential confounding effects on phosphene measures.

For assessment of visual hallucinations, the hallucinations subscale of the Neuropsychiatric Inventory $\left(\mathrm{NPI}^{\text {hall }}\right)^{33}$ was used with specific reference to the occurrence of visual hallucinations in the previous month, with the derived NPI ${ }^{\text {hall }}$ score (frequency $\times$ severity of hallucinations) subsequently used in analyses. For reliability purposes, patients were asked independently from carers about the occurrence of visual hallucinations in the month prior to TMS using screening questions derived from the North East Visual Hallucinations Inventory III; ${ }^{34}$ any discrepancies between carer/family member and patient accounts of hallucinations were discussed with both parties and the assessor, with reformulation of $\mathrm{NPI}^{\text {hall }}$ test scores (with primacy given to the opinion of the caregiver).

\section{TMS}

Testing took place in a semi-darkened room and participants wore an eye mask and were asked to keep their eyes closed during stimulations (study duration approximately $1 \mathrm{~h}$ ). To avoid the potential influence of light adaptation on the phosphene threshold, ${ }^{24}$ at $15 \mathrm{~min}$ intervals, participants were exposed to daylight-equivalent levels of luminance for at least $3 \mathrm{~min}$. The TMS was applied using a handheld $70 \mathrm{~mm}$ figure of eight coil connected to two monophasic MagStim 2002 stimulators via an intergated Bistim2 unit (MagStim Co, Dyfed, Wales). Paired pulse occipital TMS across a range of interstimulus intervals (ISI, for example between 2 and $50 \mathrm{~ms}$ ) has been demonstrated to facilitate phosphene perception above single pulse stimulation (see for example Kammer \& Baumann, ${ }^{35}$ Sparing et al ${ }^{36}$ ). In particular, Sparing et $\mathrm{al}^{36}$ observed with ISIs of between 3 and $12 \mathrm{~ms}$ and a conditioning stimulus set at $90 \%$ of the test stimulus, that normal healthy participants perceive a higher number of phosphenes to paired pulse compared with single pulse stimuli (factor increase of 1.6-1.8); in their study an ISI of $3 \mathrm{~ms}$ appeared to be no different from $12 \mathrm{~ms}$ statistically although numerically an ISI of $3 \mathrm{~ms}$ seemed slightly more facilitatory. On this basis, in the present study, to maximise phosphene response rate, we applied TMS using a paired-pulse paradigm with the conditioning stimulus intensity set at $90 \%$ of the test stimulus with an interstimulus interval of $3 \mathrm{~ms}$ and with intervals at least $10 \mathrm{~s}$ between paired pulses.

The TMS coil was applied within a surface latex grid taped to the occiput of the participant. The grid had $8 \times 81-\mathrm{cm}$-spaced points and was centred on the $\mathrm{Oz}$ point $(10 \%$ of the nasion-inion distance above the inion). The centre of the coil was placed on the intersections of the grid, with the coil handle in the midline. Current flow in the coil was craniocaudal. Nine grid intersection sites were initially assessed for phosphenes in $2 \mathrm{~cm}$ steps from $2 \mathrm{~cm}$ to left of midline to $2 \mathrm{~cm}$ to the right and $2 \mathrm{~cm}$ above $\mathrm{Oz}$ to $2 \mathrm{~cm}$ below. Mapping of stimulation sites was done in a pseudo-random order to avoid serial order effects. 


\section{Determination of phosphene threshold parameters}

Participants initially had a period of time to become accustomed to the stimuli and familiar with reporting phosphenes. At each mapped grid site the phosphene threshold was determined by increasing the stimulus intensity from $50 \%$ in a stepwise fashion in $5 \%$ (relative to maximum stimulator output) increments up to $100 \%$ of the stimulator output and decreasing the stimulus intensity in $1 \%$ steps if phosphenes were elicited (stimulus delivery maximum of $0.2 \mathrm{~Hz}$ ). Participants were asked to report the location (ipsilateral, contralateral, central or bilateral), colour and phenomenology of any visual or other subjective sensations after each stimulus. Four stimuli were given at each stimulation intensity and the lowest stimulus intensity required to elicit at least one phosphene was defined as the phosphene threshold. This lower threshold $(P=0.25$ rather than $P=0.50)$ was defined to ensure that the number of participants who might not display responses (i.e. had phosphene thresholds approaching or at $100 \%$ of stimulator output) was minimised.

As a control, sham stimulation was also given and randomly interspersed with real stimulations (1:8 sham to real stimuli at a stimulation intensity of between 100 and $120 \%$ of phosphene threshold or $100 \%$ stimulator power if phosphene threshold was not established). Sham stimulation involved tilting the coil away from the head but with one winding remaining in contact with the scalp.

In a subset of participants (10 controls and 9 patients with DLB) in whom phosphenes could be reliably reproduced and who were willing to tolerate extended study, a stimulationresponse protocol at the map site with the lowest phosphene threshold (see point (a) below) was applied. Eleven stimulation intensity levels were defined around this threshold (five above and five below in $2 \%$ increments of maximum stimulator output) for each participant and ten stimuli were given at each level (random order) and the frequency of phosphene elicitation at each stimulation intensity level was recorded.

\section{Data analysis}

Two TMS outcome measures were analysed to assess visual cortex excitability.

(a) Phosphene threshold: the threshold at the optimal stimulation site for participants who demonstrated phosphenes. It is established that given the stimulation intensity limits of TMS, not all individuals will experience phosphenes even at the maximum stimulation output (see for example Kammer et $a l,{ }^{14}$ Cowey \& Walsh, ${ }^{15}$ Silvanto et al, ${ }^{16}$ Kammer et al, ${ }^{17}$ Boroojerdi et al, ${ }^{24}$ Gerwig et al, ${ }^{25}$ Kammer \& Baumann, ${ }^{35}$ Sparing et $\left.a l^{36}\right)$. Therefore to ensure inclusion of all participants in analyses, participants who did not display phosphenes up to $100 \%$ stimulator output (i.e. had thresholds above $100 \%$ ) were arbitrated a phosphene threshold of $101 \%$.

(b) Phosphene response rate: this is the number of mapped sites (with each site stimulated up to $100 \%$ of the stimulator output) at which phosphenes can be elicited (out of a maximum of nine).

Brain atrophy occurs in dementia with Lewy bodies, although whether it affects the occipital lobe is controversial. ${ }^{37}$ Nevertheless we used structural magnetic resonance scan data to control for possible differences in occipital atrophy between the patient and control groups since for every millimetre the surface cortex is away from the stimulating coil, approximately an additional 3\% of the maximum power output is required to induce an equivalent level of brain stimulation at the motor cortex. ${ }^{38}$ Left and right occipital poles were defined in standard Montreal Neurologic Institute
(MNI) space and these points were transformed into each participant's raw image space using SPM 8 for Windows (Statistical Parametric Mapping, London). The nearest scalp points (left and right) were defined; a line between each of the brain points and their corresponding scalp points provided the long axis for $1 \mathrm{~cm}$ diameter cylinders. Non-brain volume was calculated and divided by the cross-sectional area of the respective cylinders, to provide averaged (left and right) scalp-to-occipitalsurface distances $\left(\right.$ scalp-occipital ${ }_{d}$ ). Repeat analyses were then performed using an adjusted phosphene threshold ratio (phosphene threshold/scalp-occipital d $_{\text {) }}$ to account for possible group differences in atrophy.

Primary correlation analyses assessed the relationship between $\mathrm{NPI}^{\text {hall }}$ in patients and phosphene parameters. Secondary analyses examined the association between key demographic/disease factors (age, visual acuity, UPDRS motor subscale score, CAMCOG score, visuoperceptual score and CAF score) and phosphene parameters in controls and patients where appropriate.

Parametric and non-parametric statistics were applied according to normality of the data using SPSS 17 for Windows. All values reported are means and standard deviations unless otherwise stated.

\section{Results}

\section{Demographic characteristics}

The patients with DLB and the controls were similar in terms of age, gender and visual acuity (Table 1). As expected, UPDRS motor scores were significantly higher and cognitive test and visuoperceptual task scores significantly lower in the patients compared with the controls. The distribution of $\mathrm{NPI}^{\text {hall }}$ scores for patients was positively skewed (median 3, range 1-8) with a significant proportion (7 patients) having a NPI hall score of 1 ; $\mathrm{NPI}^{\text {hall }}$ data were consequently analysed non-parametrically.

\section{Phosphene characteristics}

All participants tolerated the study and TMS reliably elicited phosphenes in a similar proportion of controls and patients (17/ 19 (90\% of controls), 17/21 ( $82 \%$ of patients); Fisher exact test, $P=0.66$ ). Sham stimulation produced phosphenes in only two participants (one control on one of the sham stimulations, one patient on six different sham stimulations). Of note, when the coil was held more anteriorly (over motor cortex) and fired, neither of these participants reported any phosphenes.

Phosphenes occurred predominately in either the lower contra-lateral hemifield or near the fovea. The subjective characteristics of the phosphenes experienced are shown in Table 2. Six patients but no controls experienced seeing transient complex figures and objects after TMS (Fisher exact test, $P=0.02$ ) with some overlap between the phenomenology of these and the individual's visual hallucination history (Appendix). In patients who experienced these visual hallucination-like phosphenes (Appendix), they occurred infrequently $(<5 \%$ of stimuli administered) between the more common classic phosphenes and were unrelated to stimulation intensity. In addition these patients had higher NPI ${ }^{\text {hall }}$ scores (median 5.0 $\quad(\mathrm{IQR}=3.0)$ compared with those who did not (median 2.0 (IQR =3.0); Mann-Whitney $U$-test 17.5, $P=0.03$ ) and worse visuoperceptual scores (median $22.5(\mathrm{IQR}=17.3)$ v. median $34.0(\mathrm{IQR}=8.0)$; $U=20.0, P=0.05)$. There was also a trend for these patients with DLB to have lower phosphene threshold scores (median 65.0 $(I Q R=29.8))$ compared with those who did not experience visual hallucination-like phosphenes (median $80.0(\mathrm{IQR}=41.0)$ ) and also higher phosphene response rate (median 8.0 $(\mathrm{IQR}=3.0) \mathrm{v}$. 


\begin{tabular}{|c|c|c|c|}
\hline & Controls $(n=19)$ & Patients $(n=21)$ & $P$ \\
\hline Age, years: mean (s.d.) & $77.6(7.1)$ & $80.6(5.9)$ & 0.15 \\
\hline Gender, males:females & $11: 8$ & $12: 9$ & 0.96 \\
\hline Unified Parkinson's Disease Rating Scale motor subscale, mean (s.d.) & $0.9(1.6)$ & $34.3(15.2)$ & $<0.001$ \\
\hline Mini-Mental State Examination, mean (s.d.) & $29.0(1.2)$ & $19.1(5.4)$ & $<0.001$ \\
\hline \multicolumn{4}{|l|}{ Cambridge Cognitive Examination, mean (s.d.) } \\
\hline Total score & $96.5(3.4)$ & $65.8(15.1)$ & $<0.001$ \\
\hline Executive subscore & $22.1(3.4)$ & $10.6(5.1)$ & $<0.001$ \\
\hline Memory subscore & $23.6(2.1)$ & $15.5(4.2)$ & $<0.001$ \\
\hline Visual acuity, decimalised & $0.64(0.29)$ & $0.56(0.25)$ & 0.36 \\
\hline Visuoperceptual score, \% correct: mean (s.d.) & $39.8(0.4)$ & $30.1(8.2)$ & $<0.001$ \\
\hline Clinician Assessment of Fluctuation, mean (s.d.) & - & $8.4(4.2)$ & N/A \\
\hline One Day Fluctuation Assessment Scale, mean (s.d.) & - & $5.6(4.6)$ & N/A \\
\hline On cholinesterase inhibitors, yes:no & - & $16: 5$ & $\mathrm{~N} / \mathrm{A}$ \\
\hline On anti-Parkinsonian medication, yes:no & - & $6: 15$ & N/A \\
\hline L-dopa dose equivalent, mg:b mean (s.d.) & - & $262.5(173.4)$ & N/A \\
\hline
\end{tabular}

median $4.0(\mathrm{IQR}=8))$ although this did not reach significance (phosphene threshold: $U=23.5, P=0.10$; phosphene response rate, $U=23.0, P=0.09$ ).

\section{Comparison of phosphene parameters across groups}

As expected there was a negative correlation between phosphene threshold and phosphene response rate (i.e. lower threshold, more sites demonstrating phosphenes) in both controls and patients (controls: Kendall's $\tau=-0.67, P<0.001$; patients: $\tau=-0.69$, $P<0.001)$. There were no significant differences between the controls and patients for phosphene threshold (controls: median $65 \% \quad(\mathrm{IQR}=44 \%)$; patients: median $72 \% \quad(\mathrm{IQR}=41 \%)$ $U=177.5, P=0.56)$ and phosphene response rate (controls: median 4.0 (IQR = 8.0); patients: median $6.0 \quad(\mathrm{IQR}=7.5)$; $U=178.5, \quad P=0.57$, Fig. 1). Stimulus response plots were comparable for controls and patients (Fig. 2); phosphene thresholds were similar between controls and patients included in the stimulation response paradigm (controls: $55.6 \%$ (s.d. $=8.8 \%$ ); patients: $59.4 \%$ (s.d. $=12.1 \%$ ); $t=0.69, P=0.50$ ) and there were no significant differences in phosphene frequency between the controls and patients across the 11 stimulation intensities used (mean difference patients minus controls: $1.0 \%$ (s.d. $=7.6 \%), F=0.21, P=0.65$ ).

Despite no differences between the controls and patients with regard to phosphene parameters and stimulus response plots, in the patients there was a negative correlation between $\mathrm{NPI}^{\text {hall }}$ score and phosphene threshold $(\tau=-0.49, P=0.005$, Fig. 3(a)) and a positive association with phosphene response rate $(\tau=0.61$, $P=0.001$, Fig. 3(b)).

Given the relationship between NPI ${ }^{\text {hall }}$ score and phosphene excitability, we retrospectively analysed whether or not the relative complexity of phosphenes experienced by both controls and patients was associated with the phosphene parameters; a phosphene complexity score was defined with one point given for each subjective feature of the phosphenes elicited in a given participant: simple phosphene, two-dimensional geometric shape, single colour (other than white/grey), polychromatic, moving phosphene, and any complex visual hallucination-like phosphene, for example figures/objects. Thus scores could potentially range from zero to six. Phosphene complexity scores were similar for both controls and patients (controls: median 1.0 $(\mathrm{IQR}=2.0)$; patients: median $1.0(\mathrm{IQR}=3.0) ; U=178, P=0.56)$ and they showed a negative correlation with phosphene threshold (controls: $\tau=-0.54, P=0.003$; patients: $\tau=-0.67, P<0.001$, Fig. $3(\mathrm{c})$ ) and a positive correlation with phosphene response rate (controls: $\tau=0.49, P=0.009$; patients: $\tau=0.55, P=0.002$, Fig. $3(\mathrm{~d}))$. In patients, the phosphene complexity score also correlated positively with NPI ${ }^{\text {hall }}$ score $(\tau=0.49, P=0.006)$.

\section{Association of phosphene parameters and NPI ${ }^{\text {hall }}$ scores with key demographic/disease factors and medication}

In both controls and patients, increasing age was negatively associated with phosphene response rate (controls: $\tau=-0.40$, $P=0.02$; patients: $\tau=-0.36, \quad P=0.04$ ) although not with phosphene threshold but there was no association between the phosphene parameters and visual acuity. In the patients there was no association between phosphene parameters and CAMCOG, UPDRS or visuoperceptual scores although there was a positive correlation between CAF scores and phosphene response rate $(\tau=0.37, P=0.03)$.

The NPI ${ }^{\text {hall }}$ scores were positively associated with CAF scores ( $\tau=0.42, P=0.02$ ) although not with other demographic/disease factors (age, visual acuity, UPDRS motor subscale score, CAMCOG score and visuoperceptual score). There were no significant differences in phosphene threshold or phosphene

\begin{tabular}{|c|c|c|c|}
\hline & $\begin{array}{c}\text { Controls, } \\
n(\%)\end{array}$ & $\begin{array}{c}\text { Patients, } \\
n(\%)\end{array}$ & $P$ \\
\hline $\begin{array}{l}\text { Participants in whom } \\
\text { phosphenes were elicited }\end{array}$ & $17 / 19(89.5)$ & 17/21 (80.9) & 0.67 \\
\hline \multicolumn{4}{|l|}{ Phosphene characteristics } \\
\hline Simple & $17 / 17(100.0)$ & 17/17 (100.0) & 1.00 \\
\hline 2D geometric shape & 4/17 (23.5) & $7 / 17$ (41.2) & 0.46 \\
\hline Coloured & $7 / 17$ (41.2) & $9 / 17(52.9)$ & 0.49 \\
\hline Polychromatic & $3 / 17(17.6)$ & $7 / 17(41.2)$ & 0.26 \\
\hline Moving & $1 / 17(5.9)$ & $0 / 17(0.0)$ & 1.00 \\
\hline Visual hallucination-like & $0 / 17(0.0)$ & 6/17 (35.3) & 0.02 \\
\hline
\end{tabular}

a. Comparison of groups on basis of chi-squared or Fisher exact tests where appropriate. Results in bold are statistically significant. 

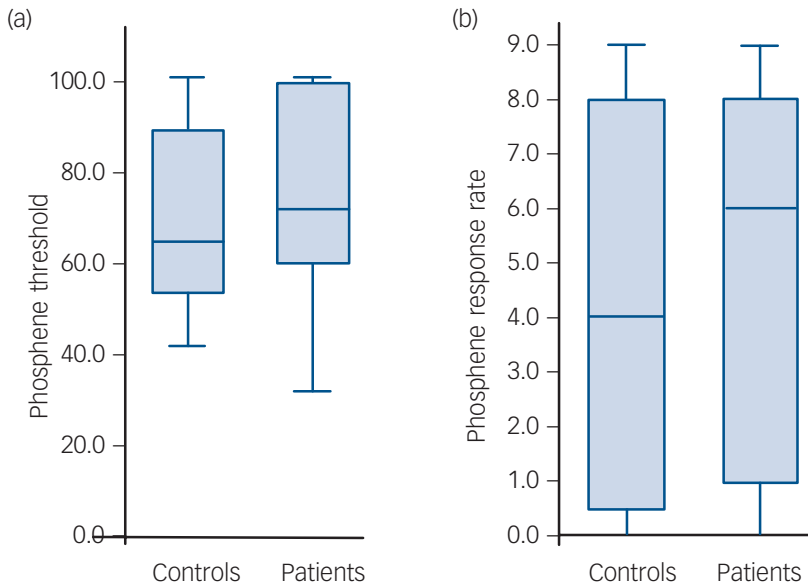

Fig. 1 Boxplots showing phosphene parameters in all participants (minimum, first quartile, median, third quartile and maximum displayed)

(a) Phosphene threshold boxplot - there were no significant differences between the controls and patients for phosphene threshold $(P=0.56)$. (b) Phosphene response rate boxplot - there were no significant differences between controls and patients for phosphene response rate $(P=0.57)$.

response rate in patients on or off cholinesterase inhibitors or anti-Parkinsonian drugs. Correlational analyses of phosphene parameters against NPI hall score restricted to patients taking cholinesterase inhibitors (phosphene threshold: $\tau=-0.63$, $P=0.002$; phosphene response rate: $\tau=0.67, P=0.001$ ) were consistent with the overall group relationships.

Use of anti-Parkinsonian medications did not significantly alter NPI ${ }^{\text {hall }}$ scores although patients on cholinesterase inhibitors appeared to have higher NPI ${ }^{\text {hall }}$ scores (on cholinesterase inhibitors, median 4.0 ( $\mathrm{IQR}=4.5$ ); not on cholinesterase inhibitors, median $1.0(\mathrm{IQR}=1.0), U=14.0, P=0.03)$. This may have been because the cholinesterase inhibitors were being used to treat visual hallucinations in dementia with Lewy bodies, as is currently recommended practice. A prior history of rapid eye movement (REM) sleep behaviour disorder in patients (11/19) was not associated with increased NPI ${ }^{\text {hall }}$ score nor any difference in phosphene parameters. It is important to note that these were exploratory analyses uncorrected for multiple comparisons and thus significant findings should be treated with caution.

\section{Influence of scalp-occipital distance on phosphene threshold}

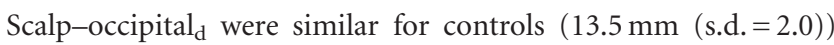
and patients $(14.3 \mathrm{~mm}$ (s.d. $=2.3) ; t=1.16, P=0.25)$. There were no significant differences between controls and patients (controls: median $5.41 \% / \mathrm{mm}(\mathrm{IQR}=2.87)$; patients: median $5.39 \% / \mathrm{mm}$ $(\mathrm{IQR}=3.08) ; U=145.0, P=0.62)$ using an adjusted phosphene threshold to account for scalp distance (phosphene threshold/ scalp-occipital ${ }_{d}$.

\section{Discussion}

This is the first study that we are aware of that uses TMS to investigate cortical visual excitability in dementia with Lewy bodies. Phosphenes were elicited reliably in the majority of controls and patients with DLB and this is likely to be related to the paired pulse paradigm ${ }^{36}$ and threshold methodology applied. Age-related effects (most studies are in young healthy adults) on

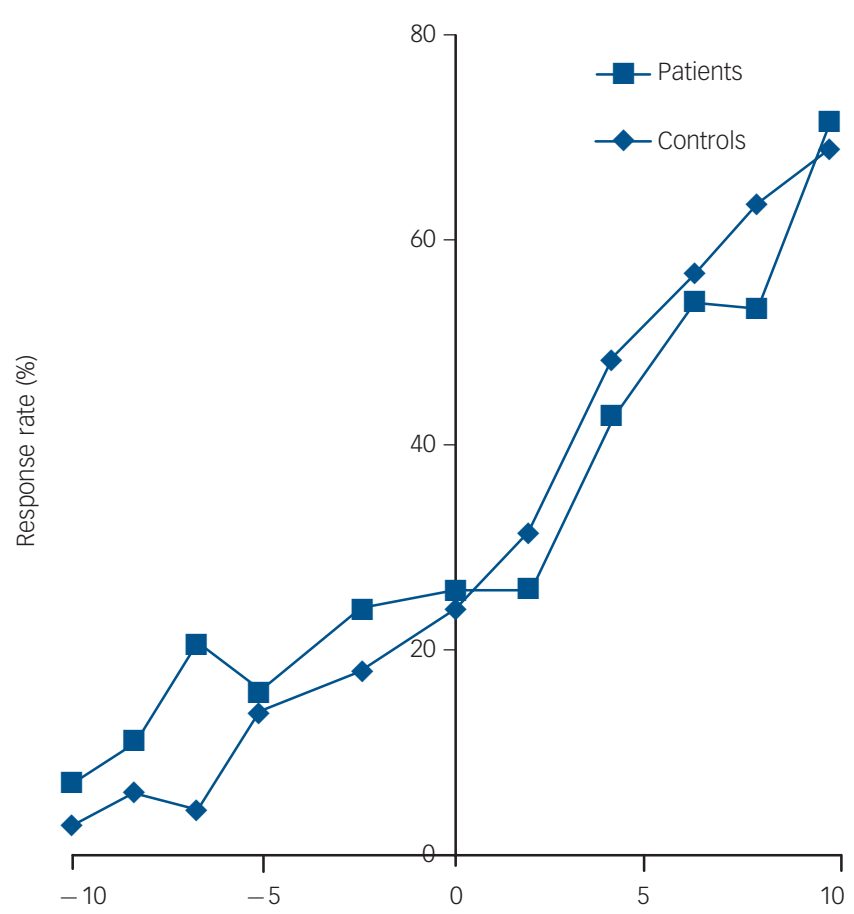

Stimulation intensity (\% of stimulator output) relative to phosphene threshold

Fig. 2 Stimulus response plot averaged across subgroups of controls $(n=10)$ and patients with dementia with Lewy bodies $(n=9)$ who underwent the stimulus response paradigm.

The $Y$-axis shows response rate expressed as a percentage of responses (out of 10 ) at a given stimulation intensity. The $x$-axis shows stimulation intensity expressed as a $\%$ of stimulator output relative to phosphene threshold.

phosphene perception are not known, although analogous analysis of motor cortical excitability has suggested an increase in thresholds with age. ${ }^{39}$ Part of this effect is presumed to be mediated by increasing scalp-to-brain-surface distances. In the present study there was no relationship between phosphene threshold and the scalp-to-occipital-cortex distance in either controls or patients, suggesting that this was unlikely to have been a factor contributing to the phosphene threshold values observed.

\section{Phosphene characteristics}

In age-matched controls, the visual characteristics of the induced phosphenes were comparable with those described in previous studies. ${ }^{14-17,24,25,35,36}$ In addition we found that: (a) phosphenes could be elicited from the majority of patients with DLB; (b) phosphene thresholds and phosphene response rates were not significantly different between groups; and (c) the input-output characteristics on stimulation response plots were comparable in the controls and patients tested. The results suggest that, first, the process of phosphene induction in controls and patients with DLB is similar and, second the structural neuronal integrity of the visual cortex activated by TMS is likely to be intact in people with dementia with Lewy bodies, which is consistent with the relative paucity of neuropathology in the occipital lobe. ${ }^{23}$

However, our findings do not support our first hypothesis that we would see increased visual cortical excitability in our patients compared with controls. Nevertheless, we found evidence to support our second hypothesis that there would be a positive correlation between the degree of visual cortical excitability as evidenced using TMS and the severity and frequency of visual 
(a)

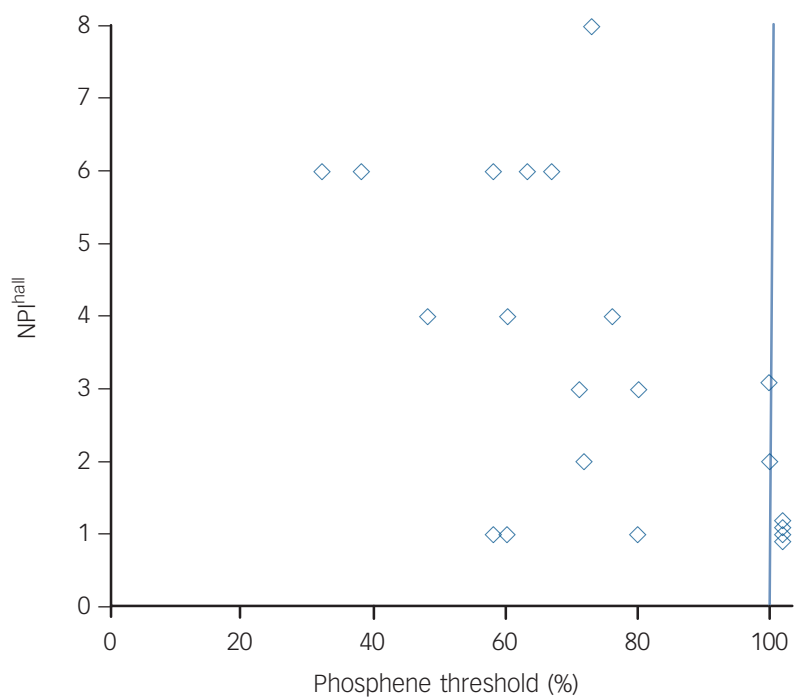

(b)

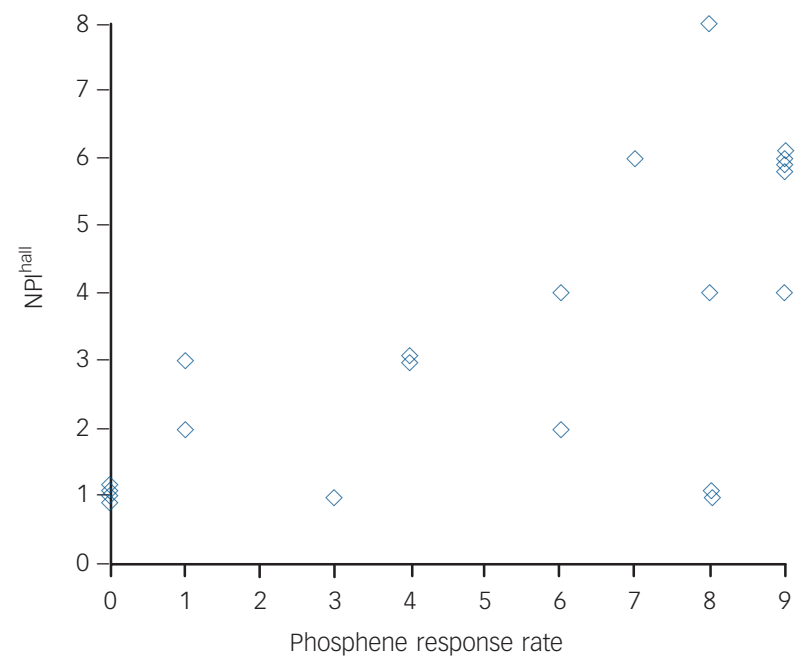

(c)

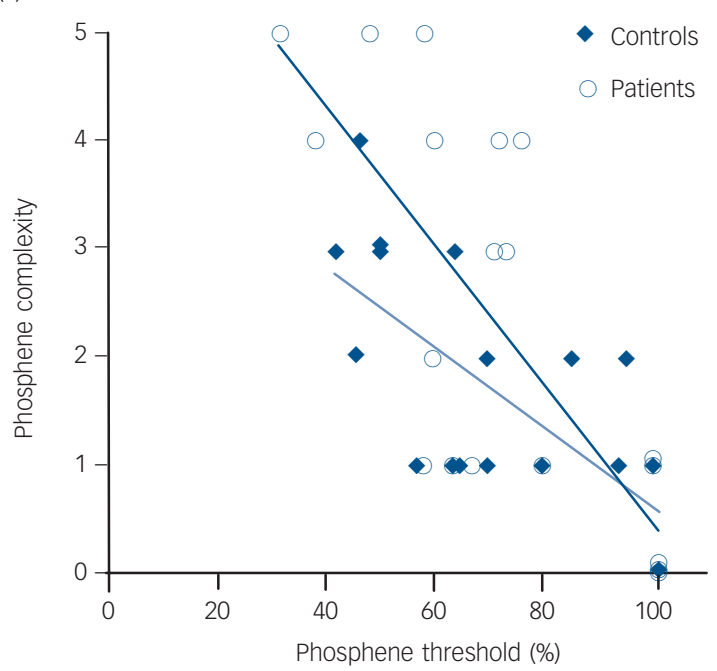

(d)

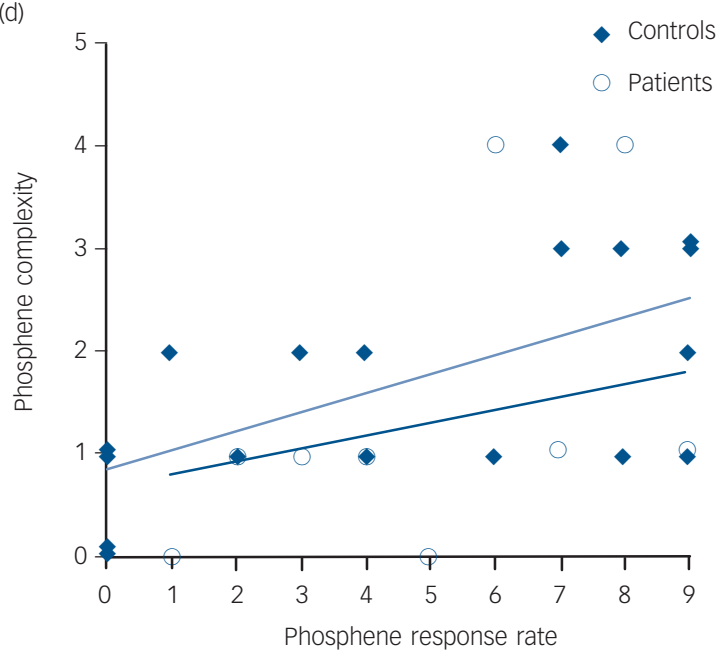

Fig. 3 Scatter plots showing phosphene parameters in patients with dementia with Lewy bodies (DLB) against Neuropsychiatric Inventory (NPI $\left.{ }^{\text {hall }}\right)$ score ((a) and (b)). (c) and (d) show phosphene parameters in all participants against phosphene complexity scores.

(a) $\mathrm{NPI}^{\text {hall }} V$. phosphene threshold: significant negative correlation $(\tau=-0.49, P=0.005)$; (b) NPI ${ }^{\text {hall }} V$. phosphene response rate: significant positive correlation $(\tau=0.61$, $P=0.001)$. (c) Complexity score $v$. phosphene threshold: controls and patients showed a negative correlation with phosphene threshold (controls: $\tau=-0.54, P=0.003$; patients: $\tau=-0.67$; $P<0.001$ ); (d) complexity score $v$. phosphene response rate: controls and patients showed positive correlation with phosphene response rate (controls: $\tau=0.49, P=0.009$. patients: $\tau=0.55, P=0.002$ ). Vertical line in (a) demarcates maximum stimulation intensity; four patients had thresholds $>100 \%$. Lines in (c) and (d) show linear fits to data (dark blue: controls; light blue: patients).

hallucinations experienced. How might we reconcile the lack of difference between the groups in terms of visual cortical excitability yet explain this association between the phosphene parameters and visual hallucinations in the patients with DLB?

One explanation may be that the patients with DLB are bimodal with regard to their visual cortical excitability. Certainly in some patients with DLB the visual cortex could be more disconnected from excitatory drive (either top-down or bottomup) as a result of pathological changes (for example, within the white matter). In others, compensatory mechanisms mediated by, for example, cholinergic receptor upregulation ${ }^{22}$ or loss of feedback/feedforward inhibition, may predominant, producing a contrary hyperexcitability state that predisposes such individuals to visual hallucinations. However, we did not see any clear dichotomy in the distribution of phosphene thresholds of the patients; indeed the spreads of distribution for the phosphene parameters were remarkably similar between controls and patients (Fig. 4).
An alternative explanation is that the phosphene parameters are inherent to the individual's 'premorbid' neurobiology and that differing levels of intrinsic visual cortical excitability contribute to the predilection in some individuals towards visual hallucinations when they develop pathological changes in the visual system. Certainly there is evidence in healthy individuals of significant differences in visual processing activity in relation to phosphene response/non-response to occipital TMS. ${ }^{40}$ However, this is a highly speculative argument that cannot be adequately tested within the present study and longitudinal studies examining the evolution and worsening of visual hallucinations in individuals with visual hallucination-prone diseases and any changes (or lack of) in phosphene parameters would be needed. In addition, a causal relationship between visual cortical excitability and visual hallucination severity and whether increased excitability leads to increased visual hallucinations or vice versa cannot be determined from the present data; it is possible that there is an inverse relationship between phosphene threshold and $\mathrm{NPI}^{\text {hall }}$ score 
because visual cortical excitability is a state-related measure or marker for more severe visual hallucinations in people with dementia with Lewy bodies.

Two other significant findings were, first, the observation that a significant minority of patients with DLB who had more marked visual hallucinations experienced visual hallucination-like phosphenes such as people or objects, and second, that there was a clear association between the complexity of phosphenes experienced and increased excitability to TMS in both controls and patients.

The origin of phosphene induction and its conscious perception although interrelated are unlikely to be co-localised, with the latter dependent upon the integrity of higher-order networks and extensive recurrent processing. ${ }^{41}$ The complexity of phosphenes elicited supports this by suggesting that localised occipital TMS leads to activation of higher visual areas. Thus it is likely that individuals with inherently increased excitability to occipital TMS have either a progressive increase in the propagation of TMS-evoked activity from early visual areas to higher areas or enhanced phosphene perception within higher visual areas themselves.

There was a trend for the patients in our study to have a greater phosphene complexity for a given phosphene threshold/ phosphene response rate compared with the controls (Fig. 3(c) and (d)) and this may suggest that there is a baseline shift in the responsiveness of higher visual processing areas to inputs from earlier visual areas either as a result of pathological hyperexcitability in these areas or a loss of inhibition. Thus in those patients with an inherently more active/excitable early visual cortex, TMS may occasionally elicit visual hallucination-like phosphenes and predispose them overall to visual hallucinations. The inverse association between phosphene threshold and NPI ${ }^{\text {hall }}$ score and the observation of possible lower phosphene threshold in people who experienced visual hallucination-like phosphenes would support this. In addition the phenomenology of the visual hallucination-like phosphenes (people and objects) experienced could indicate abnormal ventral stream/temporal lobe activation, which has been proposed as a mechanism for the production of visual hallucinations. ${ }^{6}$

However, both imaging and post-mortem studies have demonstrated that there are pathological occipital white matter changes and disruption to visual association areas in dementia

(a)

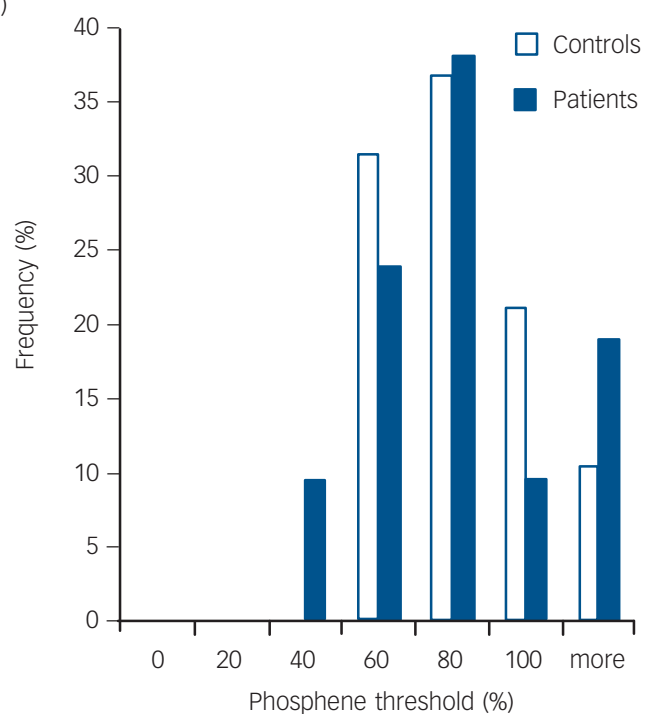

with Lewy bodies. ${ }^{12,13}$ Therefore, alternatively, the visual hallucination-like phenomena may be arising as an intrinsic misinterpretation of the induced phosphenes at higher visual processing levels as a result of impaired bottom-up transmission. As discussed previously, there is evidence for impairment in bottom-up processing in people with Parkinson's disease with visual hallucinations ${ }^{9}$ and the contribution of bottom-up processing impairment in visual hallucination manifestation would be consistent with the observation that patients with DLB frequently have illusionary experiences and marked visuoperceptual errors to normal external visual stimuli. ${ }^{28}$ Furthermore the role of impaired bottom-up processing is supported, in our study, by the higher frequency of occurrence of visual hallucination-like phosphenes in the patients with DLB with poor visuoperceptual task performance.

It has also been hypothesed that brainstem and subcortical lesions that affect ascending cholinergic, dopaminergic and serotonergic projections and their interaction with thalamocortical, basal ganglia and corticocortical neural circuitry may lead to visual hallucinations. ${ }^{7}$ In dementia with Lewy bodies, in particular, there are changes in cholinergic function within thalamic and visual associative areas that may cause visual hallucinations; certainly the clinical observation that cholinesterase inhibitors ameliorate visual hallucinations ${ }^{42}$ would support this. Cholinesterase inhibitor use could thus be a confounder in our data; intuitively one might expect individuals on cholinesterase inhibitors to have less marked visual hallucinations and lower visual cortical excitability on TMS. However: (a) subgroup analysis of patients on cholinesterase inhibitors showed that the relationship between $\mathrm{NPI}^{\text {hall }}$ score and phosphene parameters was maintained, suggesting that treatment by these agents was not an influencing factor; (b) we found no difference in our study between patients on or off cholinesterase inhibitors with regard to the visual cortical excitability; and (c) patients not on cholinesterase inhibitors appeared to have lower $\mathrm{NPI}^{\text {hall }}$ scores (although this was not significant after correction for multiple testing). This last observation is contrary to what one might expect if these agents have a treatment benefit for visual hallucinations.

Visual hallucinations have also been postulated to be associated with sleep disturbance and dream overflow. ${ }^{5}$ The occurrence of REM sleep behaviour disorder in dementia with (b)

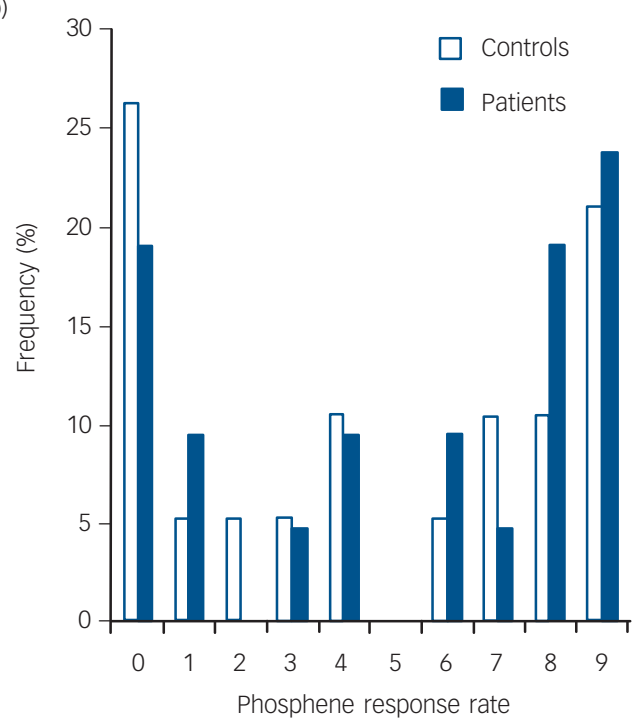


Lewy bodies is particularly frequent as a result of brainstem pathology, and in Parkinson's disease there is some evidence of an association between REM sleep behaviour disorder and the occurrence of visual hallucinations. ${ }^{43}$ However, in the present study we found no relationship between the presence/absence of REM sleep behaviour disorder and hallucination severity or, indeed, phosphene threshold or phosphene response rate.

Overall, the aetiology of visual hallucinations in dementia with Lewy bodies is likely to be distributed across multiple neural substrates $^{8}$ although mechanistically we would argue that visual cortical excitability either as a process, or a state-related measure, is central in the manifestation of visual hallucinations in dementia with Lewy bodies.

\section{Strengths and limitations}

Strengths of this study were the inclusion of individuals diagnosed according to formal diagnostic criteria and verification by consensus. In addition participants underwent detailed neuropsychological testing and a rigorous protocol for assessment of TMS-induced phosphenes. A limitation of the present study is the lack of a comparator disease group and/or a group with non-hallucinating dementia with Lewy bodies; thus it is not clear whether our findings of association between visual hallucinations and phosphene parameters are driven by the presence/absence of visual hallucinations, Lewy body-related neuropathophysiology or a general neuropathophysiological alteration associated with dementia per se.

In addition, although we found no evidence for an effect of cholinesterase inhibitors on the phosphene parameters, given the small number of patients not on cholinesterase inhibitors $(n=5)$ our study may not have been sufficiently powered to detect such a difference and thus the potential confounder effect of these medications needs further investigation.

Finally, the detection of phosphenes relied upon the subjective perception of the participant, which may be compromised by cognitive impairment, although we found no evidence for excess reporting of phosphenes in our patients, as evidenced by a lack of response to sham stimuli. Conversely, it could be argued that there may be a significant number of false-negative reports as a result of perceptual and attentional disturbances in patients with DLB and thus the true phosphene threshold is actually a lot lower in this group. Several factors, however, mitigate against this. First, the dispersion of phosphene threshold values across patients was no different from controls (Fig. 4(a)). Second, there was no correlation between decreased phosphene excitability and increased severity of cognitive fluctuations or lower cognitive scores, which one might expect if there were an association between decreased cognitive function and increased false-negative reporting.

\section{Implications}

The similarity in the phosphene parameters and their phenomenology between controls and patients with DLB suggest that the visual cortex in itself is not overtly abnormal in dementia with Lewy bodies. The positive association between excitability and visual hallucination severity suggests that the latter may be a marker for visual hallucinations, although any causal link between the two cannot be determined from the present study.

An alternative possibility to explain our findings is that the frequency and severity at which visual hallucinations are experienced may depend upon an individual's premorbid visual cortical excitability combined with subsequent pathological/ plastic changes either within the visual system or in neural systems

external to the visual system but with a significant influence on it (for example, ascending cholinergic projections). Further studies in people with non-hallucinating dementia with Lewy bodies and other dementias such as Alzheimer's are required to determine the specificity of the findings in the present paper.

In addition, studies characterising how phosphene parameters relate to the known posterior perfusion deficits in dementia with Lewy bodies and cortical visual function in early visual areas and association areas as measured by, for example, fMRI may help further elucidate the pathophysiology of visual hallucinations and visuoperceptual deficits evident in dementia with Lewy bodies. The contribution of the cholinergic system could also be examined using TMS in patients before and after cholinesterase inhibitor initiation. In addition, the use of occipital TMS, particularly if optimised to elicit visual hallucination-like phosphenes, may provide a powerful endophenotypic approach to investigating visual hallucinations in dementia with Lewy bodies and other visual hallucination-prone diseases.

\footnotetext{
John-Paul Taylor, MBBS(Hons), PhD, MRCPsych, Michael Firbank, PhD, Nicola Barnett, BSC(Hons), MSC, Sarah Pearce, MBBS, MRCPsych, Anthea Livingstone MBBS, MRCPsych, Urs Mosimann, MD, PhD, Institute for Ageing and Health, Campus for Ageing and Vitality, Newcastle University, Newcastle upon Tyne; Janet Eyre, MD, MB, ChB, BSC, DPhil, MRCPCH, Institute of Neuroscience, Newcastle University, Newcastle upon Tyne; Ian G. McKeith, MD, FRCPsych, FMedSci, John T. O'Brien, DM, FRCPsych, Institute for Ageing and Health, Campus for Ageing and Vitality, Newcastle University, Newcastle upon Tyne, UK

Correspondence: John-Paul Taylor, Institute for Ageing and Health, Newcastle University, Wolfson Research Centre, Campus for Ageing and Vitality, Newcastle upon Tyne NE4 5PL, UK. Email: john-paul.taylor@ncl.ac.uk

First received 9 Dec 2010, final revision 9 Mar 2011, accepted 16 Jun 2011
}

\section{Funding}

This work was supported by the UK NIHR Biomedical Research Centre for Ageing and Age-related disease award to the Newcastle upon Tyne Hospitals NHS Foundation Trust (RES/0211/7544), the Newcastle Healthcare Charity (BH0070250) and by the Academy of Medical Sciences and Wellcome Trust Starter Grants scheme for Clinical Lecturers (BH090112 to J-P.T.).

\section{Appendix}

\section{Subjective report of visual hallucination-like phosphenes in patients with dementia with Lewy bodies}

\section{Patient Comment during transcranial magnetic stimulation}

No. 2 'I saw a young man', 'There was a man with a round body'.

\section{No. 5 'A shadowy figure of} someone'.

No. 10 'Lots of people and children appeared'.

No. 11 'There was a person. I couldn't Seen insects and small 'black make out their features'.

No. 12 'There was a football player in a red top who then disappeared'.

No. 19 'There was a table, maybe a plank of white wood'.
Predominant phenomenology of prior visual hallucinations

Seen disembodied faces as well as figures 'all in black' and animals. Frequently reported seeing writing on walls and on furniture.

Seen children and 'shadow people'. Also reported seeing giant flowers in the garden and people in photographs 'moving' Seen children and people. lumps'.

Seen children playing and animals under tables.

Seen cats lying in bed. Also recurrently seen dead mother. 


\section{References}

1 Aarsland D, Ballard C, Larsen JP, McKeith I. A comparative study of psychiatric symptoms in dementia with Lewy bodies and Parkinson's disease with and without dementia. Int J Geriatr Psychiatry 2001; 16: 528-36.

2 Bostrom F, Jonsson L, Minthon L, Londos E. Patients with dementia with Lewy bodies have more impaired quality of life than patients with Alzheime disease. Alzheimer Dis \& Assoc Disord 2007; 21: 150-4.

3 Ricci M, Guidoni SV, Sepe-Monti M, Bomboi G, Antonini G, Blundo C, et al. Clinical findings, functional abilities and caregiver distress in the early stage of dementia with Lewy bodies (DLB) and Alzheimer's disease (AD). Arch Gerontol Geriatr 2009; 49: e101-4.

4 Collerton D, Perry E, McKeith I. Why people see things that are not there: a novel perception and attention deficit model for recurrent complex visua hallucinations. Behav Brain Sci 2005; 28: 737-57.

5 Diederich NJ, Goetz CG, Stebbins GT. Repeated visual hallucinations in Parkinson's disease as disturbed external/internal perceptions: focused review and a new integrative model. Movement Disord 2005; 20: 130-40.

6 ffytche DH, Howard RJ, Brammer MJ, David A, Woodruff P, Williams S. The anatomy of conscious vision: an fMRI study of visual hallucinations. Nat Neurosci 1998; 1: 738-42.

7 Manford M, Andermann F. Complex visual hallucinations. Clinical and neurobiological insights. Brain 1998; 121: 1819-40.

8 Archibald NK, Clarke MP, Mosimann UP, Burn DJ. The retina in Parkinson's disease. Brain 2009; 132: 1128-45.

9 Meppelink AM, de Jong BM, Renken R, Leenders KL, Cornelissen FW, van Laar T. Impaired visual processing preceding image recognition in Parkinson's disease patients with visual hallucinations. Brain 2009; 132: 2980-93.

10 Harding AJ, Broe GA, Halliday GM. Visual hallucinations in Lewy body disease relate to Lewy bodies in the temporal lobe. Brain 2002; 125: 391-403.

11 Colloby SJ, Fenwick JD, Williams ED, Paling SM, Lobotesis K, Ballard C, et al. A comparison of $(99 \mathrm{~m}) \mathrm{Tc}-\mathrm{HMPAO}$ SPET changes in dementia with Lewy bodies and Alzheimer's disease using statistical parametric mapping. Eur J NuCl Med Mol Imaging 2002; 29: 615-22.

12 Higuchi M, Tashiro M, Arai H, Okamura N, Hara S, Higuchi S, et al. Glucose hypometabolism and neuropathological correlates in brains of dementia with Lewy bodies. Exp Neurol 2000; 162: 247-56.

13 Ota M, Sato N, Ogawa M, Murata M, Kuno S, Kida J, et al. Degeneration of dementia with Lewy bodies measured by diffusion tensor imaging. NMR in Biomed 2009; 22: 280-4.

14 Kammer T, Puls K, Erb M, Grodd W. Transcranial magnetic stimulation in the visual system. II. Characterization of induced phosphenes and scotomas. Exp Brain Res 2005; 160: 129-40.

15 Cowey A, Walsh V. Magnetically induced phosphenes in sighted, blind and blindsighted observers. NeuroReport 2000; 11: 3269-73.

16 Silvanto J, Cowey A, Lavie N, Walsh V. Striate cortex (V1) activity gates awareness of motion. Nat Neurosci 2005; 8: 143-4.

17 Kammer T, Beck S, Erb M, Grodd W. The influence of current direction on phosphene thresholds evoked by transcranial magnetic stimulation. Clin Neurophysiol 2001; 112: 2015-21.

18 Gothe J, Brandt SA, Irlbacher K, Roricht S, Sabel BA, Meyer B-U. Changes in visual cortex excitability in blind participants as demonstrated by transcranial magnetic stimulation. Brain 2002; 125: 479-90.

19 Mori T, Ikeda M, Fukuhara R, Nestor PJ, Tanabe H. Correlation of visual hallucinations with occipital rCBF changes by donepezil in DLB. Neurol 2006; 66: 935-7.

20 Imamura $\mathrm{T}$, Ishii $\mathrm{K}$, Hirono $\mathrm{N}$, Hashimoto $\mathrm{M}$, Tanimukai S, Kazuai $\mathrm{H}$, et al. Visual hallucinations and regional cerebral metabolism in dementia with Lewy bodies (DLB). Neuroreport 1999; 10: 1903-7.

21 Kemp PM, Hoffmann SA, Tossici-Bolt L, Fleming JS, Holmes C. Limitations of the HMPAO SPECT appearances of occipital lobe perfusion in the differential diagnosis of dementia with Lewy bodies. NuCl Med Commun 2007; 28: 451-56

22 O'Brien JT, Colloby SJ, Pakrasi S, Perry EK, Pimlott SL, Wyper DJ, et al. Nicotinic alpha4beta2 receptor binding in dementia with Lewy bodies using
123I-5IA-85380 SPECT demonstrates a link between occipital changes and visual hallucinations. Neuroimage 2008; 40: 1056-63.

23 Gomez-Tortosa E, Newell K, Irizarry MC, Albert M, Growdon JH, Hyman BT. Clinical and quantitative pathologic correlates of dementia with Lewy bodies. Neurol 1999; 53: 1284-91.

24 Boroojerdi B, Bushara K, Corwell B, Immisch I, Battaglia F, Muellbacher W, et al. Enhanced excitability of the human visual cortex induced by short-term light deprivation. Cereb Cortex 2000; 10: 529-34.

25 Gerwig M, Niehaus L, Kastrup O, Stude P, Diener HC. Visual cortex excitability in migraine evaluated by single and paired magnetic stimuli. Headache 2005; 45: 1394-9.

26 Oliveri M, Calvo G. Increased visual cortical excitability in ecstasy users: a transcranial magnetic stimulation study. J Neurol Neurosurg Psychiatry 2003; 74: $1136-8$.

27 McKeith IG, Dickson DW, Lowe J, Emre M, O'Brien JT, Feldman $\mathrm{H}$, et al. Diagnosis and management of dementia with Lewy bodies. Third report of the DLB consortium. Neurol 2005; 65: 1863-72.

28 Roth M, Huppert FA, Mountjoy CQ, Tym E. The Cambridge Examination for Mental Disorders of the Elderly - Revised. Cambridge University Press, 1999.

29 Folstein MF, Folstein SE, McHugh PR. Mini-mental state. A practical method for grading the cognitive state of patients for the clinician. J Psychiatr Res 1975; 12: 189-98.

30 Fahn S. Unified Parkinson's disease rating scale. In Recent Developments in Parkinson's Disease: 153-63. McMillan, 1987.

31 Mosimann UP, Mather G, Wesnes KA, O'Brien JT, Burn DJ, McKeith IG. Visual perception in Parkinson disease dementia and dementia with Lewy bodies. Neurol 2004; 63: 2091-6.

32 Walker MP, Ayre GA, Cummings JL, Wesnes K, McKeith IG, O'Brien JT, et al. The Clinician Assessment of Fluctuation and the One Day Fluctuation Assessment Scale. Two methods to assess fluctuating confusion in dementia. Br J Psychiatry 2000; 177: 252-6.

33 Cummings JL, Mega M, Gray K, Rosenberg-Thompson S, Carusi DA, Gornbein J. The Neuropsychiatric Inventory: comprehensive assessment of psychopathology in dementia. Neurol 1994; 44: 2308-14.

34 Mosimann UP, Collerton D, Dudley R, Meyer TD, Graham G, Dean JL, et al. A semi-structured interview to assess visual hallucinations in older people. Int J Geriatr Psychiatry 2008; 23: 712-8.

35 Kammer T, Baumann LW. Phosphene thresholds evoked with single and double TMS pulses. Clin Neurophysiol 2010; 121: 376-9.

36 Sparing R, Dambeck N, Stock K, Meister IG, Huetter D, Boroojerdi B. Investigation of the primary visual cortex using short-interval paired-pulse transcranial magnetic stimulation (TMS). Neurosci Lett 2005; 382: 312-6.

37 Middelkoop HA, van der Flier WM, Burton EJ, Lloyd AJ, Paling S, Barber R, et al. Dementia with Lewy bodies and AD are not associated with occipital lobe atrophy on MRI. Neurol 2001; 57: 2117-20.

38 Stokes MG, Chambers CD, Gould IC, Henderson TR, Janko NE, Allen NB, et al. Simple metric for scaling motor threshold based on scalp-cortex distance: application to studies using transcranial magnetic stimulation. J Neurophysiol 2005; 94: 4520-7.

39 Rossini PM, Desiato MT, Caramia MD. Age-related changes of motor evoked potentials in healthy humans: non-invasive evaluation of central and peripheral motor tracts excitability and conductivity. Brain Res 1992; 593: 14-9

40 Meister IG, Weidemann J, Dambeck N, Foltys H, Sparing R, Krings T, et al Neural correlates of phosphene perception. In Transcranial Magnetic Stimulation and Transcranial Direct Current Stimulation. Supplements to Clinical Neurophysiology, Vol. 56 (eds W Paulus, F Tergau, MA Nitsche, JC Rothwell, U Ziemann, H Hallett): 305-11. Elsevier Science, 2003.

41 Taylor $\mathrm{P}$, Walsh $\mathrm{V}$, Eimer $\mathrm{M}$. The neural signature of phosphene perception. Hum Brain Mapp 2010; 31: 1408-17.

42 McKeith I, Del Ser T, Spano P, Emre M, Wesnes K, Anand R, et al. Efficacy of rivastigmine in dementia with Lewy bodies: a randomised, double-blind, placebo-controlled international study. Lancet 2000; 356: 2031-6.

43 Pacchetti C, Manni R, Zangaglia R, Mancini F, Marchioni E, Tassorelli C, et al Relationship between hallucinations, delusions, and rapid eye movement sleep behaviour disorder in Parkinson's disease. Mov Disord 2005; 20 : 1439-48. 